\title{
Simultaneidade de fatores de risco cardiovascular em universitários: prevalência e comparação entre períodos de graduação
}

\author{
Guilherme da Silva Gasparotto ${ }^{1}$, Elto Legnani ${ }^{2}$, Rosimeide Francisco dos Santos Legnani ${ }^{1}$, Wagner de Campos $^{3}$
}

\begin{abstract}
RESUMO
A simultaneidade de vários fatores de risco cardiovascular pode apresentar maior risco ao indivíduo do que um fator isolado. 0 objetivo deste estudo foi comparar a prevalência de fatores simultâneos entre estudantes veteranos (402) e calouros (1.197). Os fatores de risco observados, por meio de questionários foram a prática insuficiente de atividade física, os comportamentos de tabagismo, etilismo e hábitos alimentares. Mediu-se também a obesidade, o acúmulo de gordura abdominal e a pressão arterial elevada. A quantidade de fatores de risco foi categorizada quanto à freqüência: nenhum fator de risco, 1 a 3, 4 a 6 e 7 ou mais. A prevalência entre os períodos de graduação foi comparada por meio do teste do Qui-quadrado. Foi visto que a prevalência de 1 a 3 fatores de risco simultâneos foi superior entre os calouros $(39,3 \%)$ comparado aos veteranos $(25,1 \%) p<0,001$. Entre 4 e 6 fatores foi mais prevalente entre os veteranos $(67,7 \%)$ que em calouros $(55,5 \%) p<0,001$, bem como a simultaneidade de 7 ou mais fatores $(7,2 \%$ entre veteranos e $4,1 \%$ para calouros) $p<0,01$. Conclui-se que nesta população, maior quantidade de fatores de risco encontrados simultaneamente foi superior nos veteranos comparado aos calouros.
\end{abstract}

Descritores: Estudantes; Fatores de Risco; Atividade Física; Doenças Cardiovasculares.

\section{Clustering of cardiovascular risk factors in college students: prevalence and comparison between graduation periods}

\begin{abstract}
The clustering of several factors may be at greater risk to the individual than a single risk factor. The aim of this study was to compare the prevalence of clustering factors between senior students (402) and freshmen students $(1,197)$. The risk factors observed were the insufficient physical activity, the behaviors of smoking, drinking and eating habits. The obesity, abdominal fat and high blood pressure were measured. The clustering of risk factor was categorized according to the frequency, 1 to 3; 4 to 6;7 or more. The prevalences between the graduation periods were compared using the chi-square test. The prevalence of 1 to 3 clustered risk factors was higher among freshmen (39.3\%) than senior students $(25.1 \%) p<0.001$. Prevalence of 4 and 6 clustered risk factors were more among senior students $(67.7 \%)$ than freshmen $(55.5 \%) p$ $<0.001$, as well as clustered of 7 or more risk factors $(7.2 \%$ among senior students and $4.1 \%$ to freshmen) $p<0.01$. Therefore, the greatest number of clustered risk factors was found in senior students compared to freshmen.
\end{abstract}

Descriptors: Students; Risk Factors; Physical Activity; Cardiovascular Diseases.

\footnotetext{
${ }^{1}$ Mestre em Educação Física pela Universidade Federal do Paraná (UFPR), Curitiba, PR, Brasil.

${ }^{2}$ Doutor em Educação Física pela Universidade Tecnológica Federal do Paraná (UTFPR), Curitiba, PR, Brasil.

${ }^{3}$ Doutor em Educação Física pela Universidade Federal do Paraná (UFPR), Curitiba, PR, Brasil.
} 


\section{Introdução}

Quando se discute doenças cardiovasculares (DCVs) em jovens adultos, a atenção tem prioridade para os fatores de risco de desenvolvimento deste tipo de patologia, pois a presença propriamente de doenças como infarto agudo do miocárdio, acidente vascular encefálico, insuficiência cardíaca, entre outras, não tem grande frequência nesta fase da vida. Segundo a Pesquisa Nacional por Amostra de Domicílio (PNAD) realizada em 2003, menos de 5\% dos indivíduos com idade inferior a 30 anos apresentavam alguma DCV1.

Aidentificação e controle de fatores de risco cardiovascular (FRC) são importantes em todas as faixas etárias, visto a relação com o desenvolvimento deste tipo de enfermidade ${ }^{2}$. Nesta perspectiva, jovens em idade universitária mostram-se relevante população de estudo, pois estes indivíduos tendem a sofrer modificações importantes no estilo de vida e hábitos de saúde neste período ${ }^{3-5}$.

Segundo estudos prévios, entre os FRC mais frequentemente observados em universitários, destacam-se os comportamentos relacionados ao consumo de tabaco, bebidas alcoólicas, hábitos alimentares inadequados e prática insuficiente de atividade física, além de outros fatores metabólicos como a obesidade e pressão arterial elevada ${ }^{6-8}$.

Alguns autores ressaltam ser mais importante do que a observação da presença dos fatores de risco isoladamente, a identificação de simultaneidade destes em um mesmo indivíduo, o que indica aumento substancial no risco de desenvolvimento de DCVs ${ }^{9,10}$.

Estudos abordando a presença de FRC em universitários são comumente vistos na literatura, no entanto a presença de simultaneidade destes fatores entre os indivíduos foi pouco explorada nesta população $0^{11,12}$. Além disto, estes estudos geralmente são conduzidos com amostras não probabilísticas, o que impede estabelecer conclusões mais precisas acerca dos resultados ${ }^{13,14}$. Devido ainda, às importantes diferenças de comportamentos entre estudantes calouros e formandos, comparar a presença destes fatores simultâneos entre estes grupos torna-se atitude relevante, o que não se observa com frequência em pesquisas anteriores ${ }^{5,6}$.

Diante do exposto, o presente estudo pretende identificar a prevalência de simultaneidade de fatores de risco cardiovascular em universitários, bem como, comparar a prevalência destes fatores agregados entre alunos ingressantes com os alunos em fase de conclusão da graduação.

\section{Metodologia}

\section{População e Amostra}

Para o cálculo amostral, se levou em consideração os 24 mil alunos matriculados nos cursos de graduação oferecidos pela Universidade Federal do Paraná (UFPR) no ano de 2011. Destes 5.059 foram alunos ingressantes e 1.582 matriculados no último ano dos respectivos cursos, totalizando uma população alvo para a pesquisa de 6.641 alunos.

Para se obter uma amostra representativa da população de universitários da UFPR, foram adotados os seguintes critérios: nível de confiança de $95 \%$, erro amostral aceitável de $3 \%$ e proporção de $50 \%$, o que implicaria em uma amostra mínima de 921 indivíduos, somadas correções por delineamento do estudo, a amostra necessária para garantir representatividade da amostra foi 1.566 estudantes.

\section{Estratificação da amostra}

A amostra foi selecionada em três estágios, inicialmente dividida proporcionalmente de acordo com o número total de alunos ingressantes (5.059) e formandos (1.482). Posteriormente, estes grupos foram divididos proporcionalmente de acordo com o número de indivíduos matriculados nas três grandes áreas de conhecimento: Ciências Humanas, Exatas e Biológicas. As áreas com matrizes curriculares similares foram somadas, assim, os alunos pertencentes aos cursos da área Tecnológica foram somados aos das ciências Exatas e os alunos pertencentes às ciências Biológicas, foram somados com os da área da Saúde. Finalmente, estes foram divididos proporcionalmente de acordo com o turno de estudo (Diurno ou Noturno). Somente a área de ciências Biológicas não apresentou representantes do período noturno, pois os cursos são ministrados em período diurno, sem aulas no período noturno.

A seleção por áreas e turno de estudo foi realizada para garantir a representação e características socioambientais particulares da população.

A partir do número de indivíduos necessário em cada estrato, realizou-se o sorteio das turmas, que foram avaliadas em sua totalidade. Nos cursos com mais de uma turma, foi realizado sorteio para escolha de apenas uma. 


\section{Instrumentos e Procedimentos}

Os fatores de risco selecionados para se verificar possível simultaneidade foram a prática insuficiente de atividade física, utilização de tabaco, consumo de bebidas alcoólicas, consumo excessivo de bebidas alcoólicas em uma única ocasião, obesidade, acúmulo de gordura abdominal e pressão arterial elevada. Estas variáveis foram escolhidas devido à ampla abordagem pela literatura e os problemas de saúde, já demonstrados, relacionados a cada uma delas ${ }^{1,2,4}$.

\section{Prática regular de atividade física}

Para obtenção do nível de atividade física foi utilizado o IPAQ (International Physical Activity Questionnaire) versão curta, validado internacionalmente para população jovem adulta e traduzido para a língua portuguesa ${ }^{16}$. Este instrumento é composto de quatro questões com subdivisões a e b, referentes à prática de atividades: Caminhada, Moderadas, Vigorosas e ao tempo em prática sedentária. As questões são compostas de itens referentes à regularidade de prática e quantidade por sessão em minutos. As classificações indicadas no instrumento são "Muito Ativo", "Ativo", "Irregularmente Ativo" e "Sedentário", no entanto pelo fato das duas primeiras classificações (Muito ativo e ativo) cumprirem as recomendações da OMS ${ }^{17}$ de 150 minutos ou mais de atividades físicas moderadas/vigorosas (AFMV), foi utilizada uma única classificação de "Ativo" para os alunos que obtiveram tempo em atividade física de acordo com o ponto de corte sugerido, assim se seguiu: Ativo: Aquele que cumpriu as recomendações de atividades moderadas e/ou vigorosas que somaram 150 minutos ou mais por semana; Insuficientemente Ativo: Aquele que não cumpriu o mínimo de 150 minutos de atividades moderadas e/ou vigorosas acumuladas na semana.

\section{Consumo de álcool, tabaco e hábitos alimentares}

Para obtenção dos comportamentos de tabagismo, etilismo e hábitos alimentares, foram utilizadas questões específicas destes comportamentos contidas no YRBS-C (Youth Risk Behavior Surveillance), desenvolvido pelo CDC (Centers for Disease Control and Prevention), previamente validado para universitários brasileiros ${ }^{18}$.

Para verificar o hábito de tabagismo, os estudantes foram questionados sobre quantos dias, dos últimos trinta, utilizaram tabaco. Foram classificados como fumantes aqueles que utilizaram tabaco pelo menos uma vez neste período.

Sobre o consumo de álcool foram questionados quantos dias, dos últimos trinta, foi consumida pelo menos uma dose de bebida alcoólica e nos últimos trinta dias quantas vezes foram consumidas cinco ou mais doses numa mesma ocasião. Foram classificados como aqueles que bebem, os que consumiram bebidas alcoólicas pelo menos uma vez neste período.

Quanto aos hábitos alimentares, os alunos foram questionados se no dia anterior à pesquisa houve o consumo de frutas, saladas verdes, vegetais, salgadinhos (chips ou similares), bolos/tortas ou doces em geral e a quantidade consumida. $O$ consumo de frutas seguiu ponto de corte de três ou mais porções por dia, esta mesma quantidade foi definida como ponto de corte para porções de saladas verdes e/ou vegetais ${ }^{19}$.

\section{Medidas Antropométricas}

As medidas antropométricas foram realizadas de acordo com a proposta de Heyward e Stolarczyk ${ }^{20}$. Foram coletadas informações de estatura e massa corporal para o cálculo do IMC, definido pela relação equivalente da massa corporal dividido pela estatura ao quadrado (IMC = massa corporal $/$ estatura ${ }^{2}$ ). Os alunos classificados com IMC $<25 \mathrm{Kg} / \mathrm{m}^{2}$, foram denominados de eutróficos e os com IMC $\geq 25 \mathrm{~kg} / \mathrm{m}^{2}$ com excesso de pesos corporal ${ }^{2}$.

A medida da circunferência da cintura foi realizada entre o ponto íleo-cristal e a última costela flutuante. Para a classificação da circunferência abdominal foram adotados os pontos de corte sugeridos pela $\mathrm{OMS}^{21}$, sendo $\geq 94 \mathrm{~cm}$ para homens e $\geq 80 \mathrm{~cm}$ para mulheres.

\section{Pressão Arterial}

A pressão arterial sistólica (PAS) e diastólica (PAD) foram mensuradas através do método auscultatório de Karotkov, no braço direito do avaliado, utilizando um esfigmomanômetro aneróide com capacidade de $300 \mathrm{mmHg}$ e variação de 2 $\mathrm{mmHg}$, postado ao nível do coração. Para a ausculta foi utilizado um estetoscópio, posicionado acima da artéria braquial, proximal e medial a fossa cubital (aproximadamente $2 \mathrm{~cm}$ acima). Os pontos de corte adotados para pressão arterial elevada foram a pressão arterial sistólica $\geq 140 \mathrm{mmHg}$, pressão arterial diastólica $\geq 90 \mathrm{mmHg}$ ou ambas acima dos valores sugerido².

O nível socioeconômico foi avaliado adotando-se a metodologia sugerida pela Associação Brasileira de Empresas de Pesquisa22 e classificados de acordo com os seguintes estratos: A, B, C, D e E. 
A presente pesquisa foi aprovada pelo Comitê de Ética em Pesquisa com seres humanos do setor de Ciências da Saúde da Universidade Federal do Paraná, estando de acordo com a resolução n. 196/1996 do Conselho Nacional de Saúde, sob o registro CEP/SD: 1043.168.10.11.

\section{Análise estatística}

Para caracterizar a amostra de acordo com os fatores sociodemográficos, bem como, identificar a prevalência dos fatores cardiovascular de risco, recorreu-se aos procedimentos da estatística descritiva (distribuição de frequência). Para análise da simultaneidade do FRCs num mesmo indivíduo, os mesmos foram categorizados da seguinte forma: os que não apresentaram FRC (0), aqueles que apresentaram apenas um FRC (1), de um a três FRC (2), de quatro a seis FRC (3) e sete ou mais FRC (4).

A comparação das prevalências dos fatores de risco simultâneos entre estudantes calouros e formandos foi analisada por meio do teste de Qui-Quadrado $(p<0,05)$. Todas as análises foram realizadas com o software SPSS 18.

\section{Resultados}

Entre os 6.541 indivíduos elegíveis para compor a amostra, foram avaliados 1.631 alunos, distribuídos entre as três áreas de estudo, ciências humanas, exatas e biológicas, que após a retirada dos questionários respondidos incorretamente ou incompletos totalizaram 1.599 alunos, sendo 1.197 calouros (74,8\%) e 402 formandos. A média de idade dos alunos foi de $20,8 \pm 3,9$, Entre os estudantes $50,7 \%$ eram do sexo feminino, $92,9 \%$ se declararam solteiros e $75 \%$ foram classificados nos estratos socioeconômicos A (24\%) e B (51,1\%). A descrição sociodemográfica da amostra está exposta na tabela 1 abaixo.

Tabela 1 - Descrição sociodemográfica da amostra

\begin{tabular}{|c|c|c|c|}
\hline Variável & Geral \% (n) & Masc. \% (n) & Fem. $\%(n)$ \\
\hline \multicolumn{4}{|c|}{ ESTADO CIVIL } \\
\hline \multicolumn{4}{|l|}{ Calouros } \\
\hline Casado & $7,1 \%(85)$ & $5,5 \%(33)$ & $8,7 \%(52)$ \\
\hline Solteiro & $92,9 \%(1112)$ & $94,5 \%(565)$ & $91,3 \%(547)$ \\
\hline \multicolumn{4}{|c|}{ Formandos } \\
\hline Casado & $7,2 \%(29)$ & $8,4 \%(16)$ & $6,1 \%(13)$ \\
\hline Solteiro & $92,8 \%(373)$ & $91,6 \%(174)$ & $93,9 \%(199)$ \\
\hline \multicolumn{4}{|c|}{ NÍVEL SOCIOECONÔMICO } \\
\hline \multicolumn{4}{|l|}{ Calouros } \\
\hline A & $24 \%(287)$ & $24,4 \%(146)$ & $23,5 \%(141)$ \\
\hline$B$ & $51,1 \%(612)$ & $51,3 \%(307)$ & $50,9 \%(305)$ \\
\hline$C$ & $22,7 \%(272)$ & $21,6 \%(129)$ & $23,9 \%(143)$ \\
\hline$D$ & $2 \%(24)$ & $2,3 \%(14)$ & $1,7 \%(10)$ \\
\hline$E$ & $0,2 \%(02)$ & $0,4 \%(02)$ & ------ \\
\hline \multicolumn{4}{|c|}{ Formandos } \\
\hline$A$ & $26,6 \%(107)$ & $27,9 \%(53)$ & $25,5 \%(54)$ \\
\hline$B$ & $50,2 \%(202)$ & $52,1 \%(99)$ & $48,6 \%(103)$ \\
\hline C & $22,4 \%(90)$ & $19,4 \%(37)$ & $25 \%(53)$ \\
\hline $\bar{D}$ & $0,8 \%(03)$ & $0,6 \%(01)$ & $0,9 \%(02)$ \\
\hline
\end{tabular}




\begin{tabular}{|c|c|c|c|}
\hline LOCAL DE RESIDÊNCIA & & & \\
\hline \multicolumn{4}{|l|}{ Calouros } \\
\hline Com pais e irmãos & $70,7 \%(846)$ & $70,9 \%(424)$ & $70,4 \%(422)$ \\
\hline Outros parentes & $6,6 \%(79)$ & $5,8 \%(35)$ & $7,3 \%(44)$ \\
\hline Esposo (a) e filhos (as) & $7 \%(84)$ & $5,7 \%(34)$ & $8,3 \%(50)$ \\
\hline Casa de estudantes (República) & $8,6 \%(103)$ & $1,5 \%(09)$ & $7,7 \%(46)$ \\
\hline Casa de família (não parentes) & $2,2 \%(26)$ & $9,5 \%(57)$ & $2,8 \%(17)$ \\
\hline Sozinho & $4,9 \%(59)$ & $6,6 \%(39)$ & $3,3 \%(20)$ \\
\hline \multicolumn{4}{|l|}{ Formandos } \\
\hline Com pais e irmãos & $64,9 \%(261)$ & $62,6 \%(119)$ & $67 \%(142)$ \\
\hline Outros parentes & $6,2 \%(25)$ & $8,42 \%(16)$ & $4,2 \%(09)$ \\
\hline Esposo (a) e filhos (as) & $7,2 \%(29)$ & $8,42 \%(16)$ & $6,1 \%(13)$ \\
\hline Casa de estudantes (República) & $9,7 \%(39)$ & $10,5 \%(20)$ & $9 \%(19)$ \\
\hline $\begin{array}{l}\text { Casa de família } \\
\text { (não parentes) }\end{array}$ & $1,2 \%(05)$ & ------ & $2,3 \%(05)$ \\
\hline Sozinho & $10,8 \%(43)$ & $10,5 \%(20)$ & $11,4 \%(23)$ \\
\hline \multirow{2}{*}{\multicolumn{4}{|c|}{$\begin{array}{c}\text { PERÍODO DE ESTUDO } \\
\text { Calouros }\end{array}$}} \\
\hline & & & \\
\hline Diurno & $74,7 \%(894)$ & $74,2 \%(444)$ & $75,1 \%(450)$ \\
\hline Noturno & $25,3 \%(303)$ & $25,8 \%(154)$ & $24,9 \%(149)$ \\
\hline \multicolumn{4}{|l|}{ Formandos } \\
\hline Diurno & $66,9 \%(269)$ & $66,8 \%(127)$ & $67 \%(142)$ \\
\hline Noturno & $33,1 \%(133)$ & $33,2 \%(63)$ & $33 \%(70)$ \\
\hline \multirow{2}{*}{\multicolumn{4}{|c|}{$\frac{\text { ÁREA DE ESTUDO }}{\text { Calouros }}$}} \\
\hline & & & \\
\hline Humanas & $38 \%(455)$ & $30,8 \%(184)$ & $45,3 \%(271)$ \\
\hline Exatas & $37,2 \%(446)$ & $53,7 \%(321)$ & $20,9 \%(125)$ \\
\hline Biológicas & $24,8 \%(296)$ & $15,5 \%(93)$ & $33,8 \%(203)$ \\
\hline \multicolumn{4}{|l|}{ Formandos } \\
\hline Humanas & $48,5 \%(195)$ & $37,4 \%(71)$ & $24,1 \%(51)$ \\
\hline Exatas & $29,3 \%(118)$ & $42,6 \%(81)$ & $17,4 \%(37)$ \\
\hline Biológicas & $22,2 \%(89)$ & $20 \%(38)$ & $58,5 \%(124)$ \\
\hline
\end{tabular}

Com relação à prevalência dos FRC, podemos destacar o número elevado de universitários que relataram baixo consumo de frutas, verduras e legumes (<3 porções no dia anterior), elevado consumo de doces (68,9\%), álcool (64,6\%) e prática de atividade física insuficiente $(54,5 \%)$. As prevalências dos fatores de risco cardiovascular apresentados pela amostra total estão expostas na tabela 2. 
Tabela 2 - Prevalências dos fatores de risco cardiovascular.

\begin{tabular}{c|c|c}
\hline Variáveis & Prevalência (n) & IC - 95\% \\
\hline AFMV<150 min/sem. & $54,5 \%(870)$ & $52,3-57,1$ \\
\hline Fumou nos últimos 30 dias & $11,4 \%(177)$ & $9,2-13,1$ \\
\hline Consumiu álcool nos últimos 30 dias & $64,6 \%(1031)$ & $62,1-67,1$ \\
\hline Consumiu álcool em excesso em 30 dias & $40,1 \%(639)$ & $37,6-42,5$ \\
\hline Consumo < 3 porções de frutas & $92,1 \%(1470)$ & $90,9-93,4$ \\
\hline Consumo <3 porções de saladas e/ou vegetais & $80,3 \%(1281)$ & $78,3-82,1$ \\
\hline Consumo de salgados & $34,7 \%(553)$ & $32,6-37,1$ \\
\hline Consumo de doces & $68,9 \%(1099)$ & $66,8-71,2$ \\
\hline Sobrepeso/ obesidade & $25,9 \%(413)$ & $23,7-28,1$ \\
\hline Circunferência de cintura elevada & $9,9 \%(158)$ & $8,5-11,3$ \\
\hline Pressão arterial elevada & $9,5 \%(152)$ & $8,1-11,2$ \\
\hline
\end{tabular}

AFMV: Atividade Física Moderado-Vigorosa; IC: Intervalo de Confiança

Dentre os dez FRC possíveis de ser apresentados pelos estudantes, foi possível observar que a presença de nenhum fator de risco ocorreu somente em sete casos, sendo estes em calouros. Estudantes que apresentaram entre um e três fatores de risco agregados foram proporcionalmente mais numerosos entre os calouros, já para as outras duas categorias, quatro a seis e sete ou mais fatores de risco agregados, foram mais prevalentes entre os alunos formandos. A comparação das prevalências, de fatores de risco agregados, entre estudantes calouros e formandos, apresentam-se na tabela 3.

Tabela 3 - Comparação das prevalências dos fatores de risco agregados, entre estudantes calouros e formandos

\begin{tabular}{|c|c|c|c|c|c|c|c|}
\hline $\begin{array}{c}\text { Fatores } \\
\text { de risco } \\
\text { agregados }\end{array}$ & $\begin{array}{l}\text { TOTAL } \\
\%(n)\end{array}$ & IC - 95\% & $\begin{array}{c}\text { CALOUROS } \\
\%(n)\end{array}$ & IC - 95\% & $\begin{array}{c}\text { FORMANDOS } \\
\%(\mathrm{n})\end{array}$ & IC - 95\% & $p$ \\
\hline $\begin{array}{l}\text { Nenhum fator } \\
\text { de risco }\end{array}$ & $0,4(7)$ & $0,1-0,8$ & $0,6(7)$ & $0,2-1,1$ & 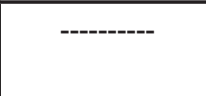 & ------- & Ns \\
\hline $\begin{array}{c}1 \text { a } 3 \text { Fatores } \\
\text { simultâneos }\end{array}$ & $35,8(572)$ & $33,3-38,1$ & $39,3(471)$ & $36,5-42,1$ & $25,1(101)$ & $21,1-29,3$ & $<0,001$ \\
\hline $\begin{array}{l}4 \text { a } 6 \text { Fatores } \\
\text { simultâneos }\end{array}$ & $58,5(936)$ & $56,2-61$ & $4,6(55)$ & $52,8-58,3$ & $67,7(272)$ & $62,9-72,1$ & $<0,001$ \\
\hline $\begin{array}{c}7 \text { ou mais } \\
\text { Fatores } \\
\text { simultâneos }\end{array}$ & $5,3(84)$ & $4,1-6,3$ & $55,5(664)$ & $3,4-5,8$ & $7,2(29)$ & $4,7-10$ & $<0,01$ \\
\hline
\end{tabular}

IC = Intervalo de Confiança; ns = não significante.

\section{Discussão}

Prevalência dos fatores de risco cardiovascular

A presença de fatores de risco cardiovascular é freqüentemente estudada em diversas populações. A importância de se observar tais fatores em populações jovens se dá justamente pela precocidade da presença destes, 0 que favorece 0 desenvolvimento de DCVs no futuro ${ }^{2,23}$.

No presente estudo $54,5 \%$ dos estudantes não cumpriram a recomendação de 150 minutos ou mais de AFMV, ou seja, mais da metade dos estudantes foram classificados como insuficientemente ativos, segundo o ponto de corte sugerido pela OMS ${ }^{17}$. 
Em vista das prevalências apresentadas em estudos similares, este valor se apresenta alto, mostrando necessidade de incentivos, políticas públicas e geração de oportunidades para prática de atividades físicas desta população $0^{5,8,24}$.

Prevalências anteriormente apresentadas, de universitários insuficientemente ativos, variam de forma importante ${ }^{5,8,24}$. Essas diferenças decorrem possivelmente, por conta da mudança de região, situação socioambiental em que se encontra a instituição estudada, instrumento e método para coleta dos dados.

A prevalência de indivíduos que consumiram cigarro nos 30 dias anteriores a pesquisa foi de 11,4\%. Estudos internacionais apresentaram prevalência de fumantes que variam de $17,6 \%$ a 28,5\%3,5,25. Em universidades brasileiras, este indicador encontra-se entre $6,3 \%$ a 21,1\% ${ }^{24,26}$. No entanto, se deve analisar estes valores com cuidado, diferenças importantes ocorrem devido ao ponto de corte adotado para classificar os fumantes. Em estudos com critério igual ao do presente, os valores foram mais próximos aos encontrados. Palma et al. ${ }^{26}$ mostraram $6,3 \%$ de fumantes e Franca e Colares ${ }^{27}$ apontaram $9 \%$.

A prevalência de estudantes que consumiram álcool nos últimos 30 dias foi de $64,8 \%$. Em estudos similares, esta variável também se apresenta elevada e frequentemente ultrapassa $50 \%$ entre aqueles que beberam. No entanto, estes trabalhos nacionais demonstram certa variação, Franca e Colares ${ }^{27}$ apontaram $59,9 \%$, já Colares et al. ${ }^{28}$ encontraram prevalência superior a estas, sendo $80,8 \%$ e Chiapetti e Serbena ${ }^{29}$ mostraram valor de $71,3 \%$ de estudantes da área da saúde que consumiram álcool no mesmo ponto de corte adotado.

O problema do consumo de bebidas alcoólicas entre universitários fica evidente quando se observa que as prevalências apresentadas são superiores aos valores divulgados pela Secretaria Nacional Antidrogas ${ }^{11}$, relativos ao consumo deste tipo de bebida entre jovens de mesma faixa etária não estudantes.

O consumo excessivo de álcool em populações universitárias tem especial importância de investigação, devido aos elevados índices deste tipo de comportamento e suas consequências, não somente para saúde cardiovascular, como também problemas sociais relacionados ${ }^{30}$. A prevalência de consumo excessivo de álcool no presente estudo foi de $40,2 \%$. Entre pesquisas que investigaram este comportamento em universitários os valores se mostraram bastante divergentes, oscilando entre $6 \%$ e 57,3\% ${ }^{24,31,32}$. Fatores como localização da instituição, quantidade de bares no entorno e ocorrências de festas estudantis podem explicar a variação deste indicador.

A ingestão insuficiente de frutas e verduras, bem como o consumo de alimentos com grande concentração de açúcar e farinha refinada, além de gorduras saturadas estão associadas à obesidade, aumento dos níveis de colesterol, resistência a insulina e outros fatores que predispõem a doenças cardiovasculares ${ }^{33,34}$. As recomendações alimentares atuais são de três ou mais porções de frutas por dia, esta mesma quantidade para o consumo de saladas e/ou vegetais, bem como a exclusão ou diminuição de alimentos riscos em carboidratos simples e alto teor de gorduras saturadas ${ }^{19}$.

A prevalência de alunos que consumiram seis ou mais porções de frutas, saladas elou vegetais somados, no dia anterior a pesquisa foi de $13,8 \%$. Vários estudos mostraram prevalências superiores, de universitários cumpridores das recomendações no consumo de frutas e vegetais, com valores que variaram de $24,9 \%$ a $35,4 \% 8,32$. No entanto, deve-se atentar que no presente estudo foi utilizada referencia proposta em 2010, de três ou mais porções de frutas e três ou mais porções de vegetais. Estudos publicados anteriormente a esta proposição, utilizaram recomendações realizadas em 1988, 1997, 1999 e 2005, onde somados frutas e vegetais a quantidade consumida deveria ser de cinco ou mais porções totais. Isto pode ter reduzido a prevalência do consumo de frutas e vegetais, comparado as apresentadas pelos estudos citados ${ }^{8,32,33}$.

Devido à praticidade de aplicação e boa associação com doenças cardiovasculares, a utilização dos índices antropométricos são frequentemente recomendados por órgãos internacionais de saúde, para estimação do sobrepeso e obesidade em diversas populações ${ }^{2}$. A utilização destes índices em universitários é comum e realizada desde décadas anteriores ${ }^{4}$. Foi possível constatar prevalência de alunos com IMC igual ou superior ao ponto de corte sugerido $\left(25 \mathrm{~kg} / \mathrm{m}^{2}\right)$ de $25,8 \%$, valor similar ao de trabalhos anteriores ${ }^{6,8,35}$. Ainda que estudos apontem resultados parecidos, deve-se atentar que mais de um quarto da população encontra-se com excesso de peso corporal.

A circunferência de cintura também é observada como importante índice preditivo de doenças cardiovasculares e sua importância já foi descrita em estudos internacionais e nacionais ${ }^{37,38}$. A prevalência de CC elevada neste estudo foi de $9,9 \%$. Em universitários mexicanos, Deschamps et al.39 encontraram prevalência de 9,5\%, enquanto no Brasil, Martins et al. ${ }^{36}$ mostraram 10,3\%.

A prevalência de pressão arterial elevada entre estudantes não é comumente observada. Sugere-se que nesta faixa etária, este indicador ainda não sofreu alterações importantes, em grande parte dos casos. Nesta pesquisa $9,5 \%$ dos alunos apresentaram pressão arterial elevada. Com exceção dos dados apresentados por Simão e colaboradores ${ }^{40}$ em estudo realizado com universitários de Lubango em Angola (20,3\%), outros trabalhos apresentaram valores entre 3,33\% e 9,7\% ${ }^{35,36,39}$. 
Simultaneidade de fatores de risco cardiovascular

Segundo Davignon e $\mathrm{Ganz}^{23}$, a presença de vários fatores de risco agregados tem relevante importância no desenvolvimento das DCVs, implicando diretamente na gênese, progressão e ocorrência dos eventos cardiovasculares futuros.

Quando se verificou a presença de poucos fatores de risco (0 e 1 a 3), a prevalência foi superior entre os calouros. De outra forma, a presença de muitos fatores num mesmo indivíduo mostrou ser mais frequente entre os formandos. Sugere-se que o período de graduação possa favorecer a aquisição de hábitos não saudáveis entre os estudantes, devido exposição superior deste grupo a eventos que podem favorecer comportamentos deletérios à saúde, além de maior envolvimento com atividades acadêmicas e extracurriculares, como estágios obrigatórios e disciplinas acumuladas durante 0 curso $0^{6,12,14}$. A alta carga de afazeres acadêmicos poderia, por exemplo, dificultar a prática regular de atividades físicas.

Esta maior ocupação, em relação aos calouros, poderia ainda, dificultar uma rotina saudável de alimentação, já que grande parte dos alunos matriculados nos últimos períodos dos respectivos cursos deve cumprir outras atividades de envolvimento acadêmico em tempo integral. Nestes casos, acabam por realizar refeições de baixo valor nutricional, pouco consumo de frutas, saladas e vegetais, além de alta ingestão de alimentos processados e com elevado teor de açúcares e gordura ${ }^{8,32}$.

O baixo nível de atividade física, associado a alimentação não saudável, favorece a condição de excesso de peso corporal e acúmulo de gordura abdominal, fatores estes que agregados a outros comportamentos, como utilização de tabaco e consumo de álcool poderiam prejudicar indicadores metabólicos, entre eles a pressão arterial².

Quanto aos hábitos de tabagismo e etilismo, alguns autores sugerem que ao ingressar na universidade, o estudante adquire sentimento de independência e necessidade de auto-afirmação frente ao novo grupo social. Diante disto, há favorecimento de aquisição deste tipo de comportamento, frente à possibilidade de aceitação entre seus pares ${ }^{3,41}$.

Segundo Rigotti e colaboradores ${ }^{42}$, alunos formandos estariam mais expostos à ambientes favorecedores de comportamento como consumo de álcool e tabaco. O maior tempo no ambiente universitário proporciona o contato com número superior de estudantes, o que aumenta as oportunidades de utilização destas substâncias. Este mesmo autor aponta que a maioria dos estudantes de uma universidade norte americana declarou ter experimentado tabaco pela primeira vez na companhia de amigos ou colegas.

O ponto forte deste estudo foi a representatividade da população estudada, com uma grande amostra subdividida proporcionalmente entre as áreas de conhecimento, calouros e formandos e turno de estudo, o que proporciona maior força para as análises. No entanto, também apresentou algumas limitações como à utilização do método recordatório para aquisição das informações relativas aos comportamentos dos estudantes. Apesar dos vieses relacionados a este tipo de metodologia, em grandes amostras, avaliações diretas tornam-se inviáveis quanto ao custo e tempo de realização deste tipo de pesquisa. Outra limitação se dá pelo ponto de corte adotado para os comportamentos de uso de tabaco (ter fumado nos últimos 30 dias) consumo de bebidas alcoólicas (ter consumido nos últimos 30 dias) e consumo insuficiente de frutas e saladas e ou verduras (no dia anterior) que não demonstram com efetividade a regularidade do comportamento. Entretanto, a presença destes comportamentos em qualquer ponto de corte nesta faixa etária demanda atenção, pois apresentam risco de continuidade ${ }^{3,4}$.

Algumas políticas institucionais poderiam amenizar esta possível diminuição da preocupação com a saúde e adesão de comportamentos não saudáveis, que ocorre entre os estudantes durante os anos de curso. Os problemas de obesidade e elevação da pressão arterial poderiam ser combatidos com o aumento de oportunidades e incentivo para prática de atividades físicas dentro e fora da universidade. Além disto, cuidados com alimentação parecem de extrema importância nesta população, visto as baixas prevalências de consumo de frutas, saladas e vegetais. Atenção especial poderia ser dada à alimentação que é disponibilizada aos estudantes fora dos restaurantes universitários, nas chamadas cantinas, onde se poderiam disponibilizar alimentos mais saudáveis como forma de lanches rápidos.

Quanto aos comportamentos de utilização de tabaco e álcool, reconhecidas as altas prevalências destes comportamentos nesta população, a realização de campanhas periódicas de conscientização quanto aos males destes hábitos seriam de extrema relevância desde a entrada dos estudantes na universidade, na tentativa de diminuir a adesão destes indivíduos a estes comportamentos.

\section{Considerações Finais}

Foi possível observar neste estudo que a simultaneidade de poucos fatores de risco é observada com maior frequência em estudantes calouros, em contrapartida, a presença de vários fatores de risco simultaneamente é mais presente em estudantes que estão prestes a terminar o período de graduação. 


\section{Referências Bibliográficas}

1. Barros MBA, Cesar CLG, Carandina L, Torre GD. Desigualdades sociais na prevalência de doenças crônicas no Brasil, PNAD-2003. Ciênc. saúde coletiva. 2006; 11(4): 911-926.

2. World Health Organization. Prevention of cardiovascular disease: Guidelines for assessment and management of total cardiovascular risk. Geneva: World Health Organization, 2007

3. Wechsler H, Rigotti NA, Gledhill-Hoyt J, Lee H. Increased Levels of Cigarette Use Among College Students. JAMA. 1998; 280(19):1-10.

4. Sahi T, Paffenbarger RS, Hsieh C, Lee IM. Body Mass Index, Cigarette Smoking, and Other Characteristics as Predictors of Self-Reported, Physician-Diagnosed Gallbladder Disease in Male College Alumni. Am J of Epidemiol. 1998; 147(7): 644-651.

5. Vankim NA, Laska MN, Ehlinger E, Story M. Understanding young adult physical activity, alcohol and tobacco use in community colleges and 4-year post-secondary institutions: A cross-sectional analysis of epidemiological surveillance data. BMC Pub Health.2010; 10: 208.

6. Desai N, Miller WC, Staples B, Bravender T. Risk Factors Associated With Overweight and Obesity in College Students. J of Am Coll Health. 2008; 57: 109-114.

7. Parker ED, Schmitz KH, Jacobs D, Dengel DR, Schreider PJ. Physical Activity in Young Adults and Incident Hypertension Over 15 Years of Follow-Up: The CARDIA Study. Am J of Pub Health. 2007; 97(4): 703-709.

8. Lowry R, Galuska DA, Fulton JE, Wechsler H, Kann L, Collins JL. Physical Activity, Food Choice, and Weight Management Goals and Practices Among U.S. College Students. Am J of Prev Med. 2000; 18(1): 18-27.

9. Legnani E, Legnani RFS, Barbosa Filho VC, Gasparotto GS Campos W, Lopes AS, . Fatores de risco à saúde cardiovascular em escolares da Tríplice Fronteira. Motriz. 2011; 17(4): 640-649.

10. Guedes DP, Guedes JERP, Barbosa DS, Oliveira JA, Stanganelli CR . Fatores de Risco Cardiovasculares em Adolescentes: Indicadores Biológicos e Comportamentais. Arq Bras Cardio. 2006; 86(6).

11. Secretaria Nacional Antidrogas. I Levantamento nacional sobre os padrões de consumo de álcool na população brasileira. SENAD, 2007.

12. Han JL, Dinger MK, Hull HR, Randal NB, Heesch KC, Fields DA. Changes in Women's Physical Activity During the Transition to College. Am J of Health Educ. 2008; 39 (4):194-199.

13. Silva LVER, Malbergier A, Stempliuk VA, et alAndrade AG. Factors associated with drug and alcohol use among university students. Rev Saúde Pub. 2006; 40(2):1-8.

14. Racette SB, Deusinger SS, Strube MJ, Highstein GR, Deusinger RH . Changes in Weight and Health Behaviors from Freshman through Senior Year of College. J of Nutr Educ Behav. 2008; 40:39-42.

15. Gasparotto GS, Gasparotto LPR, Rossi LM, Moreira NB, Bontorin MS, Campos W. Associação entre o período de graduação e fatores de risco cardiovascular em universitários. Rev Latino-Am. Enfermagem. 2013; 21(3):1-8.

16. Pardini et al. Validation of International Physical Activity Questionaire (IPAQ-6) - Pilot study with brazilians youngs adu Its. Rev Bras Cie Mov. 2001; 9(3): 45-51.

17. World Health Organization. Global recommendations on physical activity for health. Geneva: World Health Organization, 2010. 18. Teixeira M. YRBS-C: Traduction, transcultural adaptation and psicometrics propierties. Tesis of master degree, UEL, 2009.

19. U.S. Department of Agriculture and U.S. Department of Health and Human Services. Dietary Guidelines for Americans, 2010. 20. Heyward VH, Dale W. Applied Body Composition Assessment. 2a Edição, Human Kinetics, 2014.

21. World Health Organization: Preventing and Managing the Global epidemic. Geveva: World Health Organization, 1998 22. ABEP - Associação Brasileira de Empresas de Pesquisa, Critério de Classificação Econômica Brasil, www.abep.org, acessado em 26/06/2013.

23. Davignon J, Ganz P. Atherosclerosis: evolving vascular biology and clinical implications. Role of endothelial dysfunction in atherosclerosis. Circulation. 2004; 109(supll. III): 27-32.

24. Simão CB, Nahas MV, Oliveira ESA. Atividade física habitual, hábitos alimentares e prevalência de sobrepeso e obesidade em universitários da universidade do planalto catarinense - uniplac, lages SC . Rev Bras Ativ Fís Saúde. 2006. 25. Dawson KA, Schneider MA, Fletche PC, Bryden PJ. Examining gender differences in the health behaviors of Canadian university students. The J Royal Soc Promof Health. 2007; 127 (1): 38-44.

26. Palma A, Abreu RA, Cunha CA. Comportamentos de risco e vulnerabilidade entre estudantes de Educação Física. Rev Bras Epidemio. 2007; 10 (1): 117-126,. 
27. Franca C, Colares V. Estudo comparativo de condutas de saúde entre universitários no início e no final do curso. Rev Saú Púb. 2008; 42(3): 420-427.

28. Colares V, Franca C, Gonzalez E. Condutas de saúde entre universitários: diferenças entre gêneros. Cad Saúde Púb. 2009; 25(3): 521-528.

29. Chiapetti N, Serbena CA. Uso de álcool, tabaco e drogas por estudantes da área de saúde de uma Universidade de Curitiba. Psic Refl Crít. 2007; 20(2).

30. Gruenewald PJ, Johnson FW, PonickI WR, Lascala EA. A dose-response perspective on college drinking and related problems. Nat Inst Health. 2010; 105(2): 257-269.

31. Halperin AC, Smith SS, Heiligenstein E, Brown D, Fleming MF. Cigarette smoking and associated health risks among students at five universities. Nic Tob Res. 2010; 12(2): 96-104.

32. Laska MN, Pasch KE, Lust K, Story M, Ehlinger E. Latent Class Analysis of Lifestyle Characteristics and Health Risk Behaviors among College Youth. Prev Sci. 2009; 10(4): 376-386.

33. Yahia N, Achkar A, Abdallah A, Rizk S. Eating habits and obesity among Lebanese university students. Nut J. 2008; 7: 32. 34. Oviedo et al. Factores de riesgo de nfermedades crónicas no transmisibles en Estudiantes de la carrera de Medicina. Universidad de Carabobo, Venezuela. Año 2006. Nutr Hospit. 2008; 23(3):288-293.

35. Hlaing WW, Nath SD, Huffman FG. Assessing Overweight and Cardiovascular Risks Among College Students. Am J Health Educ. 2007; 38(2): $83-90$.

36. Martins et al. Pressão Arterial, Excesso de Peso e Nível de Atividade Física em Estudantes de Universidade Pública. Arq Bras Cardio. 2010; 95(2): 192-199.

37. Sarno F, Monteiro CA. Importância relativa do Índice de Massa Corporal e da circunferência abdominal na predição da hipertensão arterial. Rev Saúde Púb. 2007; 41(5): 788-796.

38. Janssen I, Katzmarzyk P, Ross R. Body Mass Index, Waist Circumference, and Health Risk. Arch Int Med. $2002 ; 162$.

39. Deschamps et al. Prevalencia de síndrome metabólico y sua sociación con el índice de masa corporal en universitários. Med Clin (Barc). 2007; 129(20): 766-9.

40. Simão M, Hayashida M, Santos CB, Cesarino EJ, Nogueira MS. Hipertensão arterial entre universitários da cidade de lubango, Angola. Rev Lat-Americana de Enf. 2008;16(4).

41. Baer JS. Student Factors: Understanding Individual Variation in College Drinking. J Study Alcohol. 2002; S. 14: 40-53.

42. Rigotti NA, Moran SE, Wechsler H. US College Students' Exposure to Tobacco Promotions: Prevalence and Association with Tobacco Use. Am J Pub Health. 95(1), 2005.

\section{Guilherme da Silva Gasparotto}

Endereço para correspondência: Rua: Coração de Maria, nº 92, Bairro: Jardim Botânico, CEP: 80210-132, Cidade: Curitiba, PR, Brasil.

E-mail: guilhermegptt@gmail.com

Lattes: http://lattes.cnpq.br/3194086558034444

Elto Legnani - eltolegnani@gmail.com

Rosimeide Franscisco dos Santos Legnani - rosilegnani@gmail.com

Wagner de Campos - wagnerufpr@ufpr.br

Enviado em 23 de julho de 2014. Aceito em 12 de março de 2015. 